\title{
Microscopic Polyangiitis with Unilateral Adrenal Hemorrhage
}

\author{
Chiharu Ito, Tetsu Akimoto, Eiji Kusano and Daisuke Nagata
}

Key words: adrenal hemorrhage, MPO-ANCA, microscopic polyangiitis

(Intern Med 53: 2023-2024, 2014)

(DOI: 10.2169/internalmedicine.53.2750)
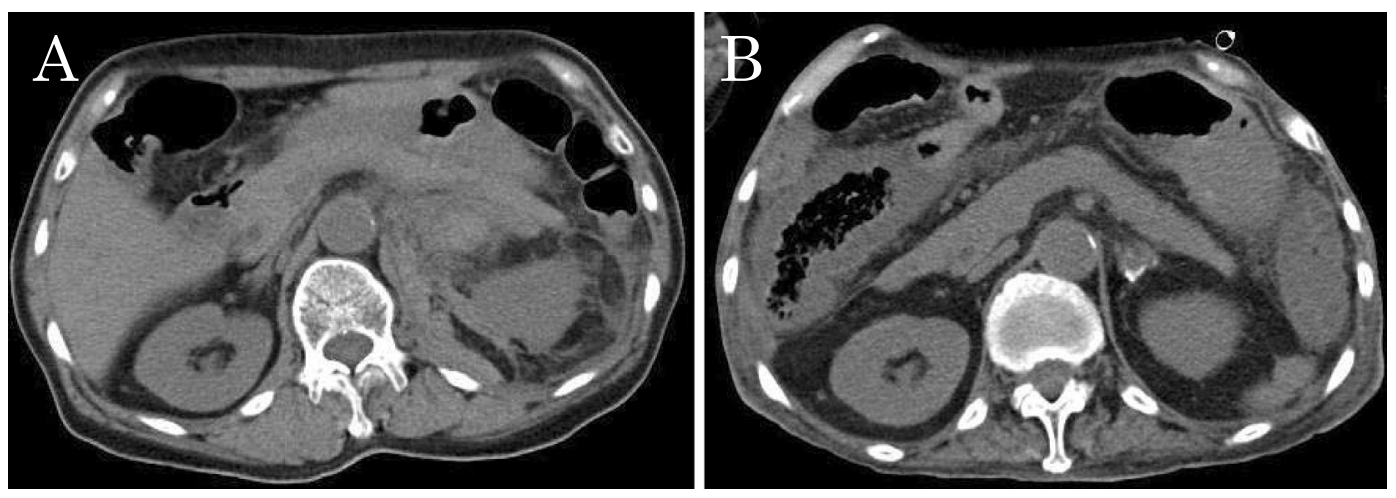

Picture 1.
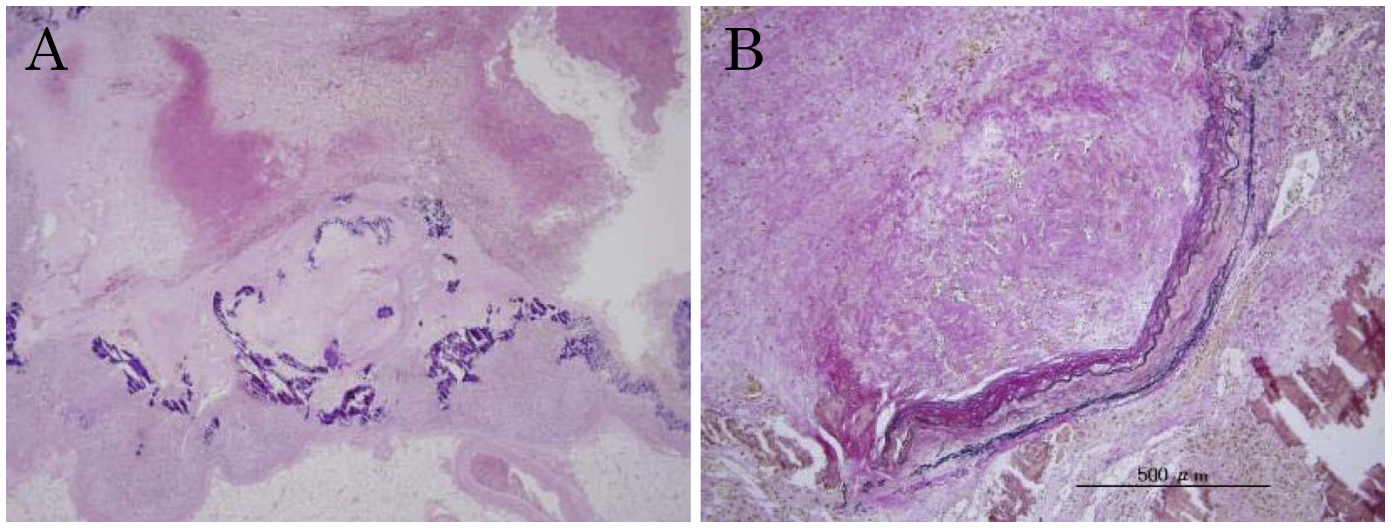

Picture 2.

A 67-year-old woman showed rapidly progressive glomerulonephritis and alveolar hemorrhage concomitant with left adrenal swelling and an increased computed tomography (CT) density of the periadrenal adipose tissue (Picture 1A). The serum MPO-ANCA titer was $364.0 \mathrm{U} / \mathrm{mL}$. Hemodialysis was introduced, and methylprednisolone semipulse therapy was initiated, followed by the administration of oral prednisolone. An abdominal CT scan taken after the initial therapy revealed that the swelling of the left adrenal gland had resolved and part of the tissue had become calcified (Picture 1B). The patient died of disseminated intravascular coagulation, and a post-mortem examination was conducted. The left adrenal medulla was necrotic, with calcification and patchy hemorrhage remaining around the periad- 
renal tissue (Picture 2A). Medium-sized healed-stage arteritis was noted (Picture 2B). Angiitis in the adrenal gland is not rare, as demonstrated in a past autopsy study (1). Adrenal hemorrhage owing to angiitis should therefore be taken into consideration while excluding other possible causes.
The authors state that they have no Conflict of Interest (COI).

\section{Reference}

1. Rott T, Vizjak A, Koselj M. ANCA-associated vasculitis: an autopsy study. Wien Klin Wochenschr 112: 671-675, 2000.

(C) 2014 The Japanese Society of Internal Medicine http://www.naika.or.jp/imonline/index.html 